\title{
Consumer Acceptability for the Patchwork Quilt Value Added Products Prepared by Quilting Technique
}

\author{
Sujata $^{1}$, K. Vasudeva Naik ${ }^{2 *}$ and Jyoti V. Vastrad ${ }^{3}$ \\ ${ }^{1}$ UAS, Dharwad, Karnataka, India \\ ${ }^{2}$ Department of Agriculture Economics, College of Agriculture, Bheemarayanagudi, \\ Karnataka, India \\ ${ }^{3} T A D$, RHSc, UAS, Dharwad, Karnataka, India
}

*Corresponding author

\section{A B S T R A C T}

\section{Keywords}

Baby quilt, Beauty, Colour, Fabric, Flourish,

Magazine holder, Quilt, Stitch, Table runner,

Technology, Tradition, Value addition

Article Info

Accepted:

10 January 2018

Available Online:

10 February 2018
The story of Indian handicrafts comes from the oldest civilizations of the world. Present study was envisaged to study the economic viability and consumer acceptability. Thirty each rural and urban consumers expressed their acceptability for patchwork quilt value added articles. Patchworks enhanced and retained the traditionality of quilt making. Both rural and urban consumers highly accepted mosaic quilt value added articles because of their striking three dimensional effect, pleasant colour combination and fibre content. Hence, the investigation is helpful to train the traditional quilt (Kaudi) makers to improve their socio-economic status.

\section{Introduction}

In order to be successful, most of the entrepreneur they need to sell their products or services.

To sell products or services consumers must accept them, which means they are willing to purchase or at least tolerate them.

Consumer acceptance is directly linked to satisfaction. Although acceptance does not guarantee satisfaction, it is a necessary step for there to be any chance of approval and repeat buying.

One major is company active in the "sensitive" products market stated: "If it was cost effective and acceptable to consumers then there would probably be an incentive to move from carton to PET because of the considerations that currently differentiate carton and PET including image to consumers and consumer acceptance, pack differentiation, robustness and effective resealability". 
In the present study, the consumer acceptability for the patchwork quilt value added articles prepared by quilting technique is elaborated;

\section{Description of the patchwork quilt value added products}

The description of the patchwork quilt value added products with respect to the blocks per patchwork pattern, number of patch patterns used and the final dimensions are recorded in Table 1. Blocks are the fabric swatches that form patchwork patterns. One basic block patchwork pattern included 9 square blocks whereas, 48 diamond swatches (blocks) were essential to prepare one unit of mosaic patchwork pattern. However, swatches in crazy patchwork had no definite pattern. Four patchwork patterns of block, mosaic and crazy techniques were used for preparation of baby quilts. For table runners three patchwork patterns of block, mosaic and crazy techniques were embedded in sashing. Table mats and magazine holders included only one patchwork pattern for value addition.

Irrespective of the type of patchwork used, the final dimensions of baby quilts was 36"x36", table runners measured 72 "x12", table mats were 19"x13" and magazine holders, 25"x20".

\section{Consumer acceptance}

Table 2 explains about the preferences of consumers for patchwork quilt value added products viz., baby quilts, table runners, table mats and magazine holders.

Among the urban consumers, 86.66 per cent of them given the first preference to mosaic patchwork baby quilt and second rank to the block patchwork baby quilt. The combination of crazy baby quilt was given third rank by 80.00 per cent of urban consumers, least preferred the traditional baby quilt. Rural consumers also had given same opinion about the baby quilt. i.e., the first rank to mosaic baby quilt (83.33\%), for crazy quilt third rank and fourth rank to the traditional baby quilt. For the table runners also consumers expressed the same rankings irrespective of their region. Eighty six per cent of rural consumers least preferred ( $4^{\text {th }}$ rank) the traditional quilt table runner. Whereas, 80.00 per cent of urban consumers highly preferred $\left(1^{\text {st }}\right.$ rank $)$ the mosaic patchwork quilt table runner. Also 76.66 per cent of urban consumers given second rank to block quilt and fourth rank to traditional quilt table runner.

Both urban (93.33\%) and rural (90.00\%) women given fourth rank to the traditional quilt table mats whereas, 86.00 per cent of rural consumers given first rank to mosaic quilt table mats and third rank to crazy quilt ones. Both rural $(83.33 \%)$ and urban $(80.00 \%)$ respondents given second rank to block quilt table mats.

Among the magazine holders, mosaic quilt gained first rank and block quilt magazine holder second rank by the 70.00 per cent of urban consumers. Traditional quilt magazine holder earned third rank followed by crazy quilt magazine holder.

As far as rural responses were considered, mosaic patchwork quilting and traditional quilt magazine holders earned first and second ranks respectively.

In general, irrespective of the rural and urban regions, all the three i.e., baby quilts, table runners and table mats has got same rankings regarding types of patchwork viz., first rank for mosaic quilt, second rank for block quilt and third rank for traditional quilting. On the contrary, the traditional quilt magazine holder got second (2.3) and third (3.1) ranks from both rural and urban consumers respectively. 
Table.1 Description of the patchwork quilt value added products

\begin{tabular}{|c|c|c|c|c|c|c|c|c|}
\hline \multirow[t]{2}{*}{$\begin{array}{l}\text { SI. } \\
\text { No. }\end{array}$} & \multirow[t]{2}{*}{ Products } & \multicolumn{3}{|c|}{ Blocks / Patchwork pattern } & \multicolumn{3}{|c|}{$\begin{array}{c}\text { Number of patchwork } \\
\text { pattern }\end{array}$} & \multirow{2}{*}{$\begin{array}{c}\text { Final } \\
\text { dimensions } \\
\text { (inches) }\end{array}$} \\
\hline & & Block (squares) & Mosaic (diamonds) & Crazy & Block & Mosaic & Crazy & \\
\hline 1. & Baby quilts & 9 & 48 & - & 4 & 4 & 4 & $36^{99} \times 36^{99}$ \\
\hline 3. & Table mat & 9 & 48 & - & 1 & 1 & 1 & $19 " \times 13 "$ \\
\hline 4. & Magazine holder & 9 & 48 & - & 1 & 1 & 1 & $25^{\prime 9} \times 20^{\prime \prime}$ \\
\hline
\end{tabular}

Table.2 Preference of the consumers for patchwork quilt value added products

\begin{tabular}{|c|c|c|c|c|c|c|c|c|c|c|c|}
\hline \multirow{2}{*}{$\begin{array}{l}\text { Sl. } \\
\text { No. }\end{array}$} & \multirow[t]{2}{*}{ Products } & \multicolumn{5}{|c|}{ Ranks by rural respondents $\mathbf{n = 3 0}$} & \multicolumn{5}{|c|}{ Ranks by urban respondents $n=30$} \\
\hline & & 1 & 2 & 3 & 4 & WAR & 1 & 2 & 3 & 4 & WAR \\
\hline I. & Baby quilts & & & & & & & & & & \\
\hline 1. & Traditional (control) & $\begin{array}{c}03 \\
(10.00)\end{array}$ & - & $\begin{array}{c}07 \\
(23.33)\end{array}$ & $\begin{array}{c}20 \\
(66.66)\end{array}$ & $3.4^{\mathrm{IV}}$ & $\begin{array}{c}01 \\
(3.33)\end{array}$ & - & $\begin{array}{c}02 \\
(6.66)\end{array}$ & $\begin{array}{c}27 \\
(90.00)\end{array}$ & $3.8^{I V}$ \\
\hline 2. & Block & $\begin{array}{c}02 \\
(6.66)\end{array}$ & $\begin{array}{c}25 \\
(83.33)\end{array}$ & $\begin{array}{c}03 \\
(10.00)\end{array}$ & - & $2.0^{\mathrm{II}}$ & $\begin{array}{c}02 \\
(6.66)\end{array}$ & $\begin{array}{c}26 \\
(86.66)\end{array}$ & $\begin{array}{c}02 \\
(6.66)\end{array}$ & - & $1.9^{11}$ \\
\hline 3. & Mosaic & $\begin{array}{c}23 \\
(76.66)\end{array}$ & $\begin{array}{c}03 \\
(10.00)\end{array}$ & $\begin{array}{c}04 \\
(13.33)\end{array}$ & - & $1.3^{\mathrm{I}}$ & $\begin{array}{c}26 \\
(86.66)\end{array}$ & $\begin{array}{c}02 \\
(6.66)\end{array}$ & $\begin{array}{c}02 \\
(6.66)\end{array}$ & - & $1.2^{1}$ \\
\hline 4. & Crazy & $\begin{array}{c}02 \\
(6.66)\end{array}$ & $\begin{array}{c}02 \\
(6.66)\end{array}$ & $\begin{array}{c}16 \\
(54.33)\end{array}$ & $\begin{array}{c}10 \\
(33.33)\end{array}$ & $2.1^{\mathrm{III}}$ & $\begin{array}{c}01 \\
(3.33)\end{array}$ & $\begin{array}{c}02 \\
(6.66)\end{array}$ & $\begin{array}{c}24 \\
(80.00)\end{array}$ & $\begin{array}{c}3 \\
(10.00)\end{array}$ & $2.9^{I I I}$ \\
\hline II. & Table runners & & & & & & & & & & \\
\hline 1. & Traditional (control) & $\begin{array}{c}01 \\
(3.33)\end{array}$ & $\begin{array}{c}03 \\
(10.00)\end{array}$ & - & $\begin{array}{c}26 \\
(86.66)\end{array}$ & $3.7^{\mathrm{IV}}$ & - & - & $\begin{array}{c}07 \\
(23.33)\end{array}$ & $\begin{array}{c}23 \\
(76.66)\end{array}$ & $3.7^{\mathrm{IV}}$ \\
\hline 2. & Block & $\begin{array}{c}07 \\
(23.33)\end{array}$ & $\begin{array}{c}19 \\
(63.33)\end{array}$ & $\begin{array}{c}02 \\
(6.66)\end{array}$ & $\begin{array}{c}02 \\
(6.66)\end{array}$ & $1.9^{\mathrm{II}}$ & $\begin{array}{c}04 \\
(13.33)\end{array}$ & $\begin{array}{c}23 \\
(76.66)\end{array}$ & $\begin{array}{c}03 \\
(10.00)\end{array}$ & - & $1.9^{11}$ \\
\hline 3. & Mosaic & $\begin{array}{c}20 \\
(66.66)\end{array}$ & $\begin{array}{c}04 \\
(13.33)\end{array}$ & $\begin{array}{c}06 \\
(20.00)\end{array}$ & - & $1.5^{\mathrm{I}}$ & $\begin{array}{c}24 \\
(80.00)\end{array}$ & $\begin{array}{c}04 \\
(13.33)\end{array}$ & $\begin{array}{c}02 \\
(6.66)\end{array}$ & - & $1.2^{1}$ \\
\hline 4. & Crazy & $\begin{array}{c}02 \\
(6.66)\end{array}$ & $\begin{array}{c}04 \\
(13.33)\end{array}$ & $\begin{array}{c}22 \\
(73.33)\end{array}$ & $\begin{array}{c}02 \\
(6.66)\end{array}$ & $2.8^{111}$ & $\begin{array}{c}02 \\
(6.66) \\
\end{array}$ & $\begin{array}{c}03 \\
(10.00)\end{array}$ & $\begin{array}{c}18 \\
(60.00)\end{array}$ & $\begin{array}{c}07 \\
(23.33) \\
\end{array}$ & $3.0^{111}$ \\
\hline
\end{tabular}


Contd...

\begin{tabular}{|c|c|c|c|c|c|c|c|c|c|c|c|}
\hline \multirow[t]{2}{*}{$\begin{array}{l}\text { Sl. } \\
\text { No. }\end{array}$} & \multirow[t]{2}{*}{ Different techniques } & \multicolumn{5}{|c|}{$\begin{array}{l}\text { Ranks by rural respondents } \\
\qquad \mathbf{n}=\mathbf{3 0}\end{array}$} & \multicolumn{5}{|c|}{$\begin{array}{l}\text { Ranks by urban respondents } \\
\qquad \mathbf{n}=\mathbf{3 0}\end{array}$} \\
\hline & & 1 & 2 & 3 & 4 & WAR & 1 & 2 & 3 & 4 & WAR \\
\hline III. & Table mats & & & & & & & & & & \\
\hline 1. & Traditional (control) & - & - & $\begin{array}{c}03 \\
(10.00)\end{array}$ & $\begin{array}{c}27 \\
(90.00)\end{array}$ & $3.9^{\mathrm{IV}}$ & - & - & $\begin{array}{c}02 \\
(6.66)\end{array}$ & $\begin{array}{c}28 \\
(93.33)\end{array}$ & $3.8^{\mathrm{IV}}$ \\
\hline 2. & Block & $\begin{array}{c}04 \\
(13.33)\end{array}$ & $\begin{array}{c}25 \\
(83.33)\end{array}$ & $\begin{array}{c}01 \\
(3.33)\end{array}$ & - & $1.9^{\mathrm{II}}$ & $\begin{array}{c}06 \\
(20.00)\end{array}$ & $\begin{array}{c}24 \\
(80.00)\end{array}$ & - & - & $1.8^{I I}$ \\
\hline 3. & Mosaic & $\begin{array}{c}26 \\
(86.66)\end{array}$ & $\begin{array}{c}04 \\
(13.33)\end{array}$ & - & - & $1.1^{\mathrm{I}}$ & $\begin{array}{c}24 \\
(80.00)\end{array}$ & $\begin{array}{c}06 \\
(20.00)\end{array}$ & - & - & $1.2^{I}$ \\
\hline 4. & Crazy & - & $\begin{array}{c}01 \\
(3.33)\end{array}$ & $\begin{array}{c}26 \\
(86.66)\end{array}$ & $\begin{array}{c}03 \\
(10.00)\end{array}$ & $3.0^{\mathrm{III}}$ & - & - & $\begin{array}{c}28 \\
(93.33)\end{array}$ & $\begin{array}{c}02 \\
(6.66)\end{array}$ & $2.8^{I I I}$ \\
\hline IV. & Magazine holder & & & & & & & & & & \\
\hline 1. & Traditional (control) & $\begin{array}{c}10 \\
(33.33)\end{array}$ & $\begin{array}{c}04 \\
(13.33)\end{array}$ & $\begin{array}{c}11 \\
(36.66)\end{array}$ & $\begin{array}{c}05 \\
(16.66)\end{array}$ & $2.3^{\mathrm{II}}$ & $\begin{array}{c}03 \\
(10.00)\end{array}$ & $\begin{array}{c}05 \\
(16.66)\end{array}$ & $\begin{array}{c}08 \\
(26.66)\end{array}$ & $\begin{array}{c}14 \\
(46.66)\end{array}$ & $3.1^{\mathrm{III}}$ \\
\hline 2. & Block & $\begin{array}{c}07 \\
(23.33)\end{array}$ & $\begin{array}{c}09 \\
(30.00)\end{array}$ & $\begin{array}{c}09 \\
(30.00)\end{array}$ & $\begin{array}{c}05 \\
(16.66)\end{array}$ & $2.4^{\mathrm{III}}$ & $\begin{array}{c}04 \\
(13.33)\end{array}$ & $\frac{21}{(70.00)}$ & $\begin{array}{c}03 \\
(10.00)\end{array}$ & $\begin{array}{c}02 \\
(6.66)\end{array}$ & $2.1^{I I}$ \\
\hline 3. & Mosaic & $\begin{array}{c}10 \\
(30.33)\end{array}$ & $\begin{array}{c}10 \\
(33.33)\end{array}$ & $\begin{array}{c}08 \\
(26.66)\end{array}$ & $\begin{array}{c}02 \\
(6.66)\end{array}$ & $2.0^{\mathrm{I}}$ & $\begin{array}{c}21 \\
(70.00)\end{array}$ & $\begin{array}{c}02 \\
(6.66)\end{array}$ & $\begin{array}{c}04 \\
(13.33)\end{array}$ & $\begin{array}{c}03 \\
(10.00)\end{array}$ & $1.6^{I}$ \\
\hline 4. & Crazy & $\begin{array}{c}03 \\
(10.00)\end{array}$ & $\begin{array}{c}07 \\
(23.33)\end{array}$ & $\begin{array}{c}2 \\
(6.66)\end{array}$ & $\begin{array}{c}18 \\
(60.00)\end{array}$ & $3.1^{\mathrm{IV}}$ & $\begin{array}{c}02 \\
(6.66)\end{array}$ & $\begin{array}{c}02 \\
(6.66)\end{array}$ & $\begin{array}{c}15 \\
(50.00)\end{array}$ & $\begin{array}{c}11 \\
(36.66)\end{array}$ & $3.1^{\mathrm{IV}}$ \\
\hline
\end{tabular}

Note:

Figures in parentheses indicate percentages

WAR: Weighted Average Ranking 
This may be due to the fibre content and colour combination used for the products. Moreover, the three dimensional effect imparted to the quilt top enhanced the aesthetic appeal making it more elegant and versatile for varied application. All the block patchwork quilt value added products were ranked second by the consumers that is mainly because of the standing star motif that was not only appealing but also could be functionally used for educating children. The bold and bright coloured blocks in patchwork pattern could be used to extend the concept of size and colour to pre-school children using indigenous resources. Traditional quilt value added products included quilt tops with random placement of swatches giving less importance to size, shape, colour, texture and fibre content. There is a need to educate the quilt makers regarding the designing and development of value added products with all the above considerations.

From the literature it is clear that for many years, traditional quilt (kaudi) making did not gain immense popularity, recognition, appreciation and it has remained only as a process of joining old left over pieces to meet the needs as poor man's blanket in the major corners of North Karnataka. It is therefore proved that, value addition to traditional quilt
(Kaudi) promotes and revives the traditional art of recycling to provide gainful employment to the tribal women and preserve cultural heritage of India. Among the patchwork quilt value added products, all the mosaic quilt value added products were highly accepted by both rural and urban consumers. Consumers also opined that if more number of items were developed through patchwork and quilting technique, that products may hold lots of market potentials and can be adapted at cottage level. Women can start a small enterprise for developing articles as per demand by forming self-help group.

\section{References}

Chaudhari, B., 1982, Tribal development in India - problems and prospects. Inter India Publications, Delhi, pp. 49-52.

https://bizfluent.com/facts-7292560-meaningconsumer-acceptability-.html

https://glosbe.com/en/en/consumer\%20accept ance

Valentine, M.D., 1973, Practical encyclopedia of crafts, Sterling publishing Company, New York, 120-126.

www.revival/finny.html

www.wikipedia.needlewok/quilting.html

\section{How to cite this article:}

Sujata, K. Vasudeva Naik and Jyoti V. Vastrad. 2018. Consumer Acceptability for the Patchwork Quilt Value Added Products Prepared by Quilting Technique. Int.J.Curr.Microbiol.App.Sci. 7(02): 1085-1089. doi: https://doi.org/10.20546/ijcmas.2018.702.135 